\title{
A novel non-thermal resection tool in endoscopic management of scarred polyps $\square$
}

\section{(ㄷ)(1) $\odot(9)$}

\author{
Authors \\ Kesavan Kandiah ${ }^{1}$, Sharmila Subramaniam ${ }^{1}$, Fergus Chedgy ${ }^{1}$, Sreedhari Thayalasekaran ${ }^{1}$, Daniel Venetz ${ }^{2}$, Patrick \\ Aepli² $^{2}$ Pradeep Bhandari ${ }^{1}$
}

Institutions

1 Portsmouth Hospitals NHS Trust, Portsmouth, United Kingdom

2 Luzerner Kantonsspital, Luzern, Switzerland

submitted 11.9.2018

accepted after revision 9.11.2018

\section{Bibliography}

DOI https://doi.org/10.1055/a-0838-5424 |

Endoscopy International Open 2019; 07: E974-E978

(c) Georg Thieme Verlag KG Stuttgart · New York elSSN 2196-9736

\section{Corresponding author}

Professor Pradeep Bhandari, Consultant Gastroenterologist and Endoscopist, Queen Alexandra Hospital, Portsmouth

PO63LY, United Kingdom

Fax: +02392286000

deep3570@yahoo.co.uk

\section{ABSTRACT}

Background and study aims Scarred polyps are challenging to resect using conventional endoscopic mucosal resection (EMR) techniques. The aim of this pilot study was to assess the feasibility of the EndoRotor device in resecting scarred polyps arising from previous endoscopic resection attempts.

Patients and methods This was a prospective pilot study of patients with scarred colonic polyps treated using EndoRotor carried out in two centers.

Results A total of 19 patients were included in this study. The overall cure rate using EndoRotor was $84 \% ; 10$ patients (52.6\%) achieved cure after one attempt and six patients (31.5\%) achieved cure after two attempts. A total of three patients who had polyp recurrence after the first EndoRotor resection were referred for either endoscopic submucosal dissection (2 patients) or surgery (1 patient) due to difficult access. There were no perforations, delayed bleeding, postpolypectomy syndrome or complications requiring surgery. Conclusions In this pilot study, the novel non-thermal device (EndoRotor) has been demonstrated to be a safe and effective technique in challenging management of scarred polyps. Further randomized controlled trials comparing this technique with APC, hot avulsion, ESD and endoscopic full-thickness resection are required to ascertain the utility of EndoRotor in the hands of non-expert endoscopists.

\section{Introduction}

Endoscopic mucosal resection (EMR) is the most widely used method for resecting colonic polyps. Although this technique is safe, it carries a risk of polyp recurrence with rates varying between $16 \%$ and $30 \%$ in polyps larger than $20 \mathrm{~mm}$ [1,2]. Polyp recurrence tends to form over a fibrosed or scarred submucosal base, making it very challenging to resect using conventional EMR techniques. Presence of fibrosis in a scarred polyp leads to lack of lift, making it difficult to catch the polyp in the snare or leading to too much of it being caught, resulting in diathermy-induced perforation.

The EndoRotor device (Interscope Medical, Inc., Worcester, Massachusetts, United States) is a novel non-thermal endo- scopic mucosal resection device (NED) for use in the gastrointestinal tract for removal of benign neoplastic tissue. It has built-in suction, which allows easy capture of non-lifting polyps and a non-thermal cutting mechanism. Initial animal studies have demonstrated the feasibility, safety and capability of this non-thermal device [3]. Subsequently, EndoRotor has been demonstrated to be feasible and safe in resection of non-dysplastic Barrett's esophagus [4]. The aim of this pilot study was to assess the feasibility of using the EndoRotor device to resect scarred colonic polyps arising from previous endoscopic resection attempts. 


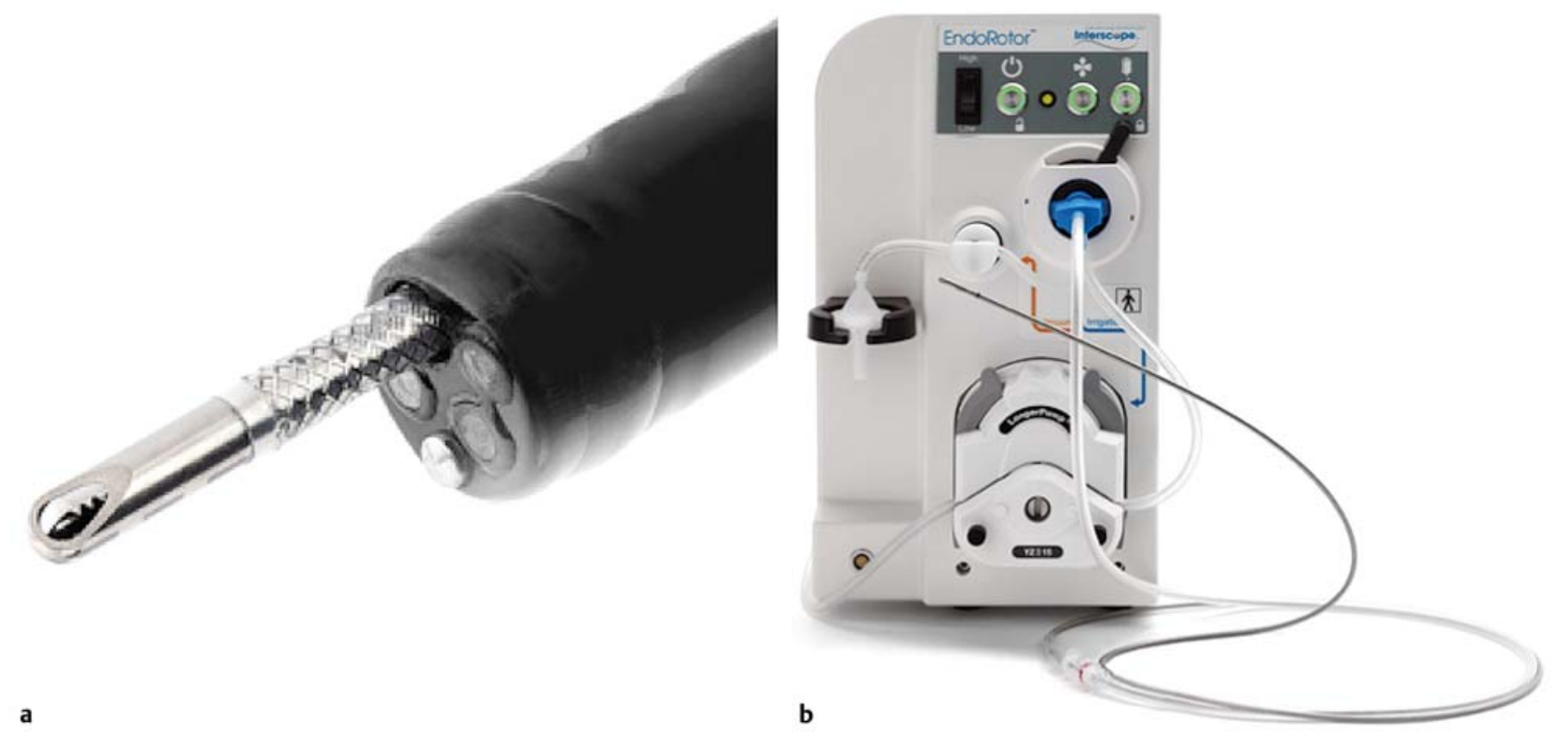

- Fig. 1 a EndoRotor catheter and b console. (Source: INTERSCOPE INC)

\section{Patients and methods}

\section{Patients}

This was a prospective pilot study of patients with scarred colonic polyps treated at two tertiary endoscopy centers (Portsmouth in United Kingdom and Lucerne in Switzerland) between 2016 and 2017. Institutional review board approval was obtained (PHT/3569). Informed consent for the procedure was obtained from all patients, and data were prospectively recorded in an electronic database. Patients received standard bowel preparation and all procedures were performed under conscious sedation by two expert endoscopists (PB and PA). Patients referred from other institutions with scarred polyps were included in this study. Scarred polyps in this study were defined as polyps with tethered bases resulting from previous attempts at resection by endoscopic mucosal resection. All patients had the rest of their colon inspected for further polyps prior to resection of their scarred polyp.

\section{Technical device}

EndoRotor is a non-thermal resection device in the form of a single-use disposable catheter that is passed through the instrument channel of an endoscope ( $\mathbf{F i g . 1}$ ). It is available in two lengths: $1890 \mathrm{~mm}$ and $1250 \mathrm{~mm}$. The outer diameter of both catheters is $3 \mathrm{~mm}$. The catheter contains a fixed outer cannula with a hollow inner cannula that rotates at either 1000 or 1700 revolutions per minute (rpm). Both the outer and inner cannulas have an orifice/opening that allows suction within the inner cannula to pull tissue into the device where the rotating inner cannula cuts it. The vacuum levels providing the suction can be varied depending on the degree of contact with the tissue. The system self-irrigates the catheter, allowing for re-

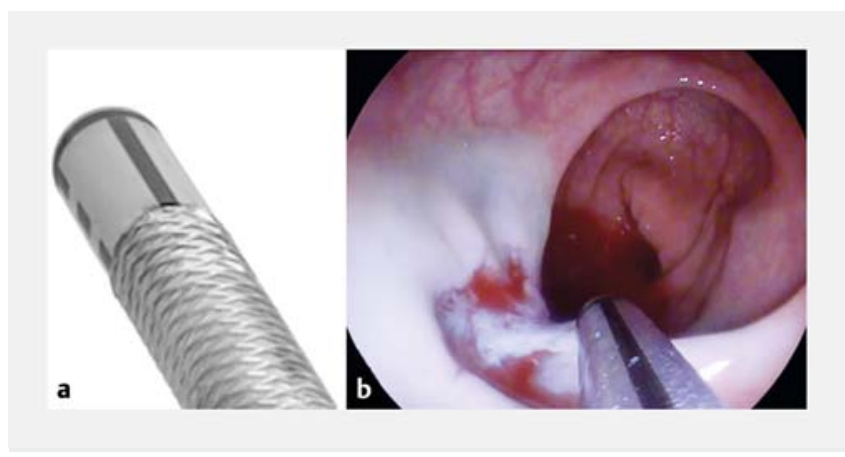

Fig. 2 Orientation of the orifice at the end of the catheter is aided by one solid black line and two interrupted black lines. a Interrupted black lines indicate that the orifice in 90 degrees away. (Source: INTERSCOPE INC) b Solid black line indicates that the orifice is 180 degrees away.

sected tissue to be transported back to a tissue trap located on the resection system.

Rotation of the inner cutting blade and suction are controlled by blue and orange foot pedals, respectively. As a safety measure, rotation of the cutting blade stops automatically after 8 seconds if suction is not continuously activated. To initiate resection, the blue pedal is pressed once and released followed by pressing the orange pedal continuously. Orientation of the cutting orifice at the end of the catheter is identified by a solid black line located 180 degrees away from the orifice ( $\triangleright$ Fig. 2 ). To terminate the resection, the orange pedal is released to deactivate the suction. Alternatively, the catheter can be lifted away from the tissue by manipulating the endoscope tip. 


\section{Non-thermal resection technique using EndoRotor}

All polyps were assessed for signs of invasion using high-definition white light and optical enhancement technology such as narrow band imaging (Olympus, Tokyo, Japan) and blue light imaging (Fujifilm, Tokyo, Japan). Scarred polyps with no evidence of invasive neoplasia were included. Although the device allows for resection without any submucosal injection, we decided to attempt injection so as to delineate a blue submucosal plane and introduce adrenaline into the polyp base. The injected lifting solution consisted of succinylated gelatine solution, indigo carmine and dilute adrenaline. The EndoRotor catheter was then inserted into the instrument channel and the solid black line at the tip of the catheter was positioned directly above the polyp. The blue pedal was pressed once to activate the rotating blade followed by continuous depression of the orange pedal, which sucked the polyp tissue into the cutting orifice. Resection of the polyp could be carried out in any direction. However, as the blade within the catheter rotates clockwise, resection was easier if the direction of resection was from the right to left. Paris 0-Is polyps were debulked using standard snare resection prior to using EndoRotor ( $\bullet$ Video 1$)$. Supplementary diathermy to the resection base was not used to avoid deep mural injury. All intraprocedural complications such as bleeding or perforation were prospectively recorded. Intraprocedural bleeding was further subcategorized as minor or major. Minor bleeding was defined as oozing from a venous vessel that did not require intervention to achieve hemostasis and no requirement for blood transfusion. Major bleeding was defined as non-spurting venous bleeds or arterial bleeds requiring endoscopic, radiological or surgical intervention to achieve hemostasis or requirement for blood transfusion.

\section{Follow-up}

The area of the non-thermal resected polyp was assessed at least 2 months following index resection with EndoRotor. The area was assessed for recurrence using high-definition white

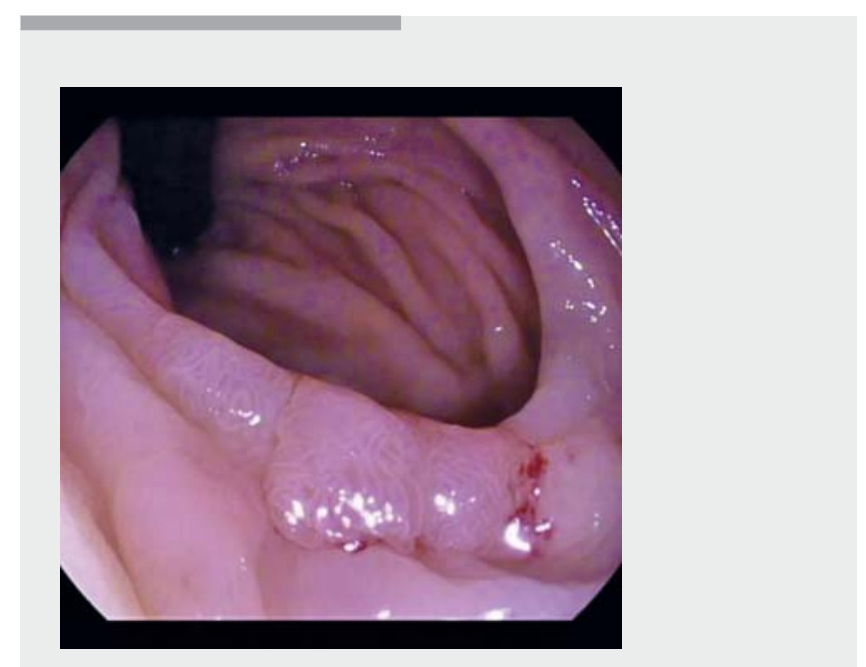

Video 1 Non-thermal resection of a scarred polyp located in the sigmoid colon using EndoRotor.

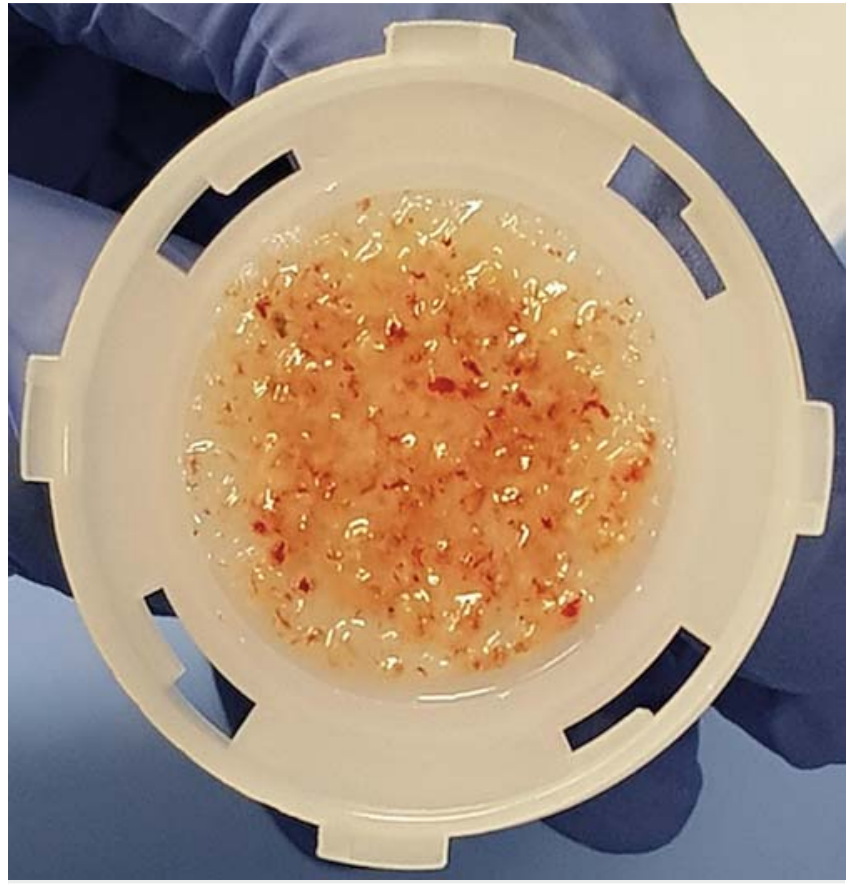

Fig. 3 Resected tissue on the filter, which is seated within the tissue trap.

light and optical enhancement technology. Biopsies from the resection margins were obtained for histological assessment for recurrence. A curative resection was achieved when there was no evidence of endoscopic or histological recurrence in the follow-up endoscopic assessment. In patients with recurrence, a further attempt at resecting the polyp using EndoRotor was undertaken at least 4 months from the date of the initial non-thermal resection.

\section{Pathology}

The resected tissue was collected in the tissue filter within a tissue trap located on the console ( $\mathbf{F i g} . \mathbf{3}$ ). The filter containing the resected tissue was then placed into a specimen pot containing formalin and sent to the histopathology laboratory. Here, the tissue specimen was processed in a similar manner as tissue obtained using standard endoscopic biopsy forceps. The size of the resections with EndoRotor was between 2 and $5 \mathrm{~mm}$, comparable to the size of tissue obtained using a standard biopsy forceps ( $\bullet$ Fig. 4 ). All dysplastic tissue was independently reviewed by two pathologists.

\section{Results}

A total of 19 patients (13 from United Kingdom and 6 from Switzerland), were included in this study. Mean age of the patients was 71 years $(50-86$ years) and $58 \%(n=11)$ were male. Median lesion size was $20 \mathrm{~mm}(7-70 \mathrm{~mm})$. All polyps were located within the rectum and sigmoid. Detailed descriptions of the resected polyps are shown in $>$ Table 1 . The overall cure rate using EndoRotor was $84 \% ; 10$ patients (52.6\%) achieved cure after one attempt and six patients (31.5\%) achieved cure after 


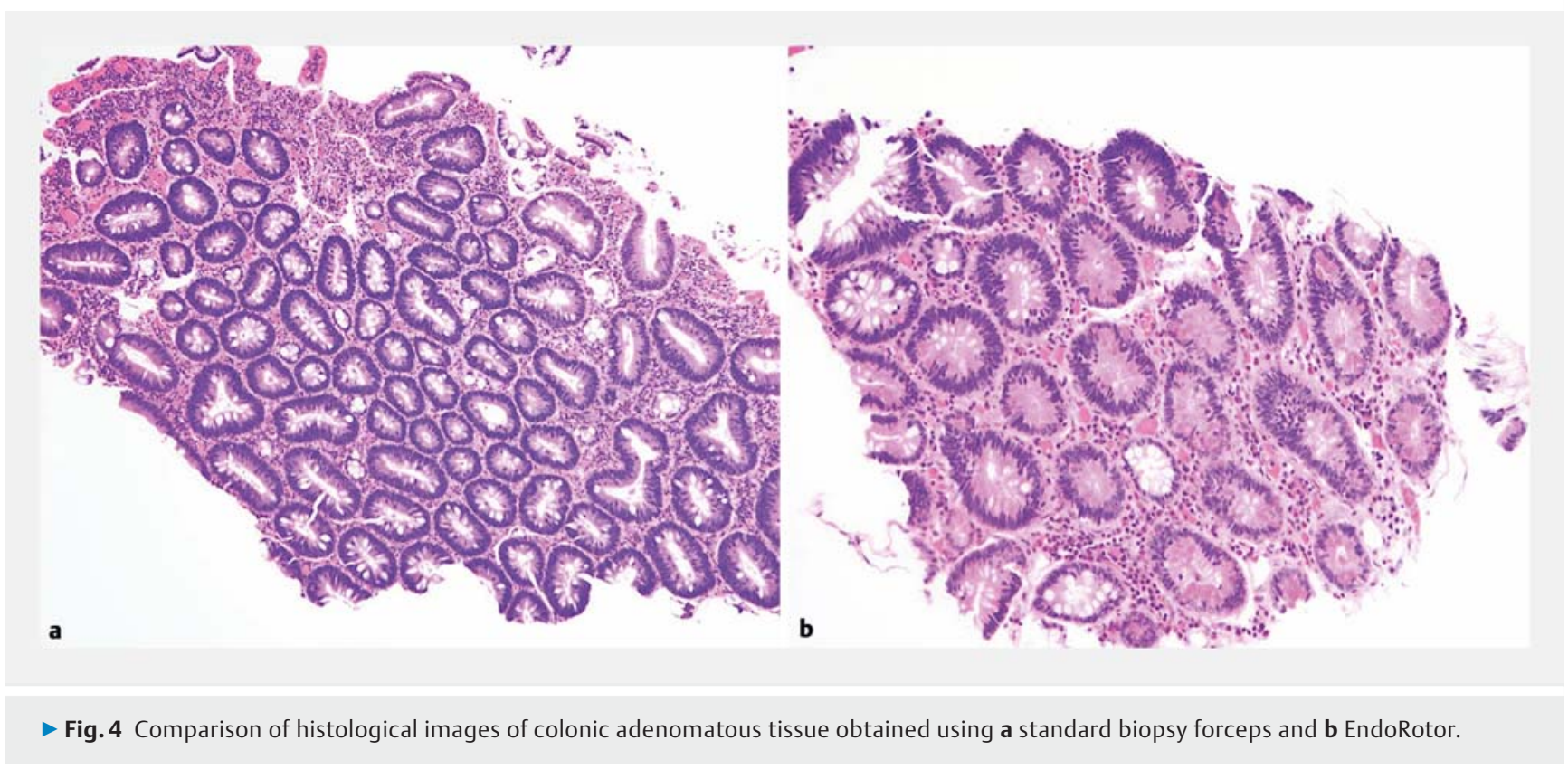

two attempts. A total of three patients who had polyp recurrence after the first EndoRotor resection were referred for either endoscopic submucosal dissection (2 patients) or surgery (1 patient) due to difficult access. Two patients experienced intraprocedural bleeding that was managed endoscopically: one was managed with bipolar hemostatic forceps and the procedure was continued to completion and the second patient was managed using endoscopic clips, thereby preventing completion of the procedure. This second patient was subsequently referred for an ESD at a later date. There were no perforations, delayed bleeding, post-polypectomy syndrome or complications requiring surgery. On logistic regression analysis, patients with difficult access were more likely to require more than one resection attempt or fail to achieve cure, albeit this did not rise to statistical significance (OR 2.00, $P=0.491$ ). All resected tissue was automatically sucked and collected in the trap. Pathologists had no concerns in reporting on these specimens.

\section{Discussion}

Endoscopic resection of scarred colonic polyps can be challenging. In this pilot study, resection of scarred polyps in the rectosigmoid colon was shown to be feasible, safe, and effective using EndoRotor. The cure rate following two resection sessions with EndoRotor was $84 \%$ in a population of patients who would have had to otherwise resort to surgery. The leading cause of polyps requiring more than one resection session or failure to achieve cure was difficult access to the polyp. We did not observe any post-polypectomy syndrome, perforation or delayed bleeding in these challenging polyps in our series, potentially due to the non-thermal characteristic of this resection device.

Several alternative techniques are currently employed to treat scarred colonic polyps. Argon plasma coagulation (APC) and avulsion using hot biopsy forceps have been used with varying success in managing scarred polyps. The drawbacks of these techniques are high recurrence rates ( $59.3 \%$ with APC and $10.3 \%$ with avulsion) and with APC, there is a lack of acquisition of tissue for histopathological assessment [5]. A new technique involving use of cold avulsion and adjuvant snare tip soft coagulation has shown promising results in management of non-lifting laterally spreading lesions with recurrence rates of $15.2 \%$ to $27.5 \%$ at 4 to 6 months [6]. However, this study was carried out in a single center and a large multicenter study would be required to assess the applicability of the technique by non-expert endoscopists. Endoscopic submucosal dissection (ESD), on the other hand, enables en bloc resection of polyps with low recurrence rates (<3\%) [7]. However, ESD is technically challenging and is associated with a high complication rate $(4 \%-20 \%)$, leading to a limited uptake of this technique in the West, especially for non-lifting scarred polyps [7]. A more recently introduced technique in resecting non-lifting polyps is endoscopic full-thickness resection using a full-thickness resection device (FTRD). The limitation of this device is that it can only be used on polyps measuring up to $30 \mathrm{~mm}$ [8].

There are disadvantages to the EndoRotor technology. The first is that it is difficult to retroflex the endoscope with the resection catheter inside the working channel. Therefore, therapy cannot be undertaken in retroflexion. The second is that the catheter requires an endoscope with a working channel of $3.2 \mathrm{~mm}$ or more, thereby limiting the choice of endoscopes that can be used with this system. The main limitation of this study is that it involved a small cohort of patients. It did not address the question of how this technique compares directly with established techniques for treating scarred polyps mentioned above. It did, however, validate a safe and effective technique in challenging management of scarred polyps. Further randomized controlled trials comparing this technique with APC, hot avulsion, ESD and endoscopic full-thickness resection are required to ascertain the utility of EndoRotor in the hands of non-expert endoscopists. 
- Table 1 Outcomes of patients treated with EndoRotor.

\begin{tabular}{|c|c|}
\hline Follow-up, median (range), months & $4(2-7)$ \\
\hline \multicolumn{2}{|l|}{ Lesion location, n (\%) } \\
\hline - Rectum & $11(58)$ \\
\hline - Rectosigmoid & $4(21)$ \\
\hline - Sigmoid & $4(21)$ \\
\hline \multicolumn{2}{|l|}{ Lesion size, n (\%) } \\
\hline - $\leq 10 \mathrm{~mm}$ & $4(21)$ \\
\hline - $11-19 \mathrm{~mm}$ & $5(26)$ \\
\hline - $\geq 20 \mathrm{~mm}$ & $10(53)$ \\
\hline
\end{tabular}

Lesion morphology, $\mathrm{n}(\%)$

\begin{tabular}{|l|r|}
\hline - Paris 0-Ila & $14(74)$ \\
\hline - 0 -Ilb & $2(10)$ \\
\hline - 0 -Is & $3(16)$ \\
\hline
\end{tabular}

Lesion predominant pit pattern, $\mathrm{n}(\%)$

\begin{tabular}{|l|r|}
\hline - IIIs & $8(42)$ \\
\hline - IIIL & $5(26)$ \\
\hline - IV & $6(32)$ \\
\hline Number of resection attempts for cure, $\mathrm{n}(\%)$ & \\
\hline - 1 & $10(52)$ \\
\hline - 2 & $6(32)$ \\
\hline Lesion histology, $\mathrm{n}(\%)$ & \\
\hline - Tubulovillous adenoma & $4(21)$ \\
\hline - Villous adenoma & $4(21)$ \\
\hline - Tubular adenoma & $11(58)$ \\
\hline Complications, $\mathrm{n}(\%)$ & \\
\hline - Perforation & $0(0)$ \\
\hline - Minor intraprocedural bleeding & $2(11)$ \\
\hline - Major intraprocedural bleeding & $0(0)$ \\
\hline - Delayed bleeding & $0(0)$ \\
\hline - Complications requiring surgery & $0(0)$ \\
\hline
\end{tabular}

Competing interests

None

References

[1] Moss A, Williams SJ, Hourigan LF et al. Long-term adenoma recurrence following wide-field endoscopic mucosal resection (WF-EMR) for advanced colonic mucosal neoplasia is infrequent: results and risk factors in 1000 cases from the Australian Colonic EMR (ACE) study. Gut 2015; 64: 57-65

[2] Knabe M, Pohl J, Gerges C et al. Standardized long-term follow-up after endoscopic resection of large, nonpedunculated colorectal lesions: a prospective two-center study. Am J Gastroenterol 2014; 109 : $183-189$

[3] Hollerbach S, Wellmann A, Meier P et al. The EndoRotor ${ }^{\circledR}$ : endoscopic mucosal resection system for non-thermal and rapid removal of esophageal, gastric, and colonic lesions: initial experience in live animals. Endosc Int Open 2016; 04: E475 - E479

[4] Knabe M, Blößer S, Wetzka J et al. Non-thermal ablation of non-neoplastic Barrett's esophagus with the novel EndoRotor ${ }^{\circledR}$ resection device. United Eur Gastroenterol J 2018; 5: 678-683

[5] Holmes I, Kim HG, Yang D-H et al. Avulsion is superior to argon plasma coagulation for treatment of visible residual neoplasia during EMR of colorectal polyps (with videos). Gastrointest Endosc 2016; 84: $822-829$

[6] Tate D, Bahin F, Desomer L et al. Cold-forceps avulsion with adjuvant snare-tip soft coagulation (CAST) is an effective and safe strategy for the management of non-lifting large laterally spreading colonic lesions. Endoscopy 2017; 50: 52-62

[7] Pimentel-Nunes P, Dinis-Ribeiro M, Ponchon T et al. Endoscopic submucosal dissection: European Society of Gastrointestinal Endoscopy (ESGE) Guideline. Endoscopy 2015; 47: 829-854

[8] Aepli P, Criblez D, Baumeler S et al. Endoscopic full thickness resection (EFTR) of colorectal neoplasms with the Full Thickness Resection Device (FTRD): Clinical experience from two tertiary referral centers in Switzerland. United Eur Gastroenterol J 2018; 6: 463-470

\section{CORRECTION}

Kandiah K, Subramaniam S, Chedgy F et al. A novel non-thermal resection tool in endoscopic management of scarred polyps

Endoscopy International Open 2019; 07: E974 - E978. DOI: $10.1055 / a-0838-5424$

In the above mentioned article a value in $>$ Table 1 under Lesion size was wrong. Correct is: $\geq 20 \mathrm{~mm}$ 